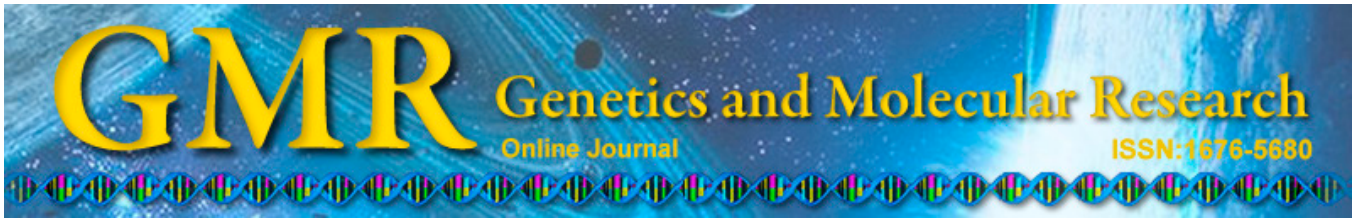

\title{
Identification of conserved microRNAs and their target genes in Nile tilapia (Oreochromis niloticus) by bioinformatic analysis
}

\author{
X.H. Li, J.S. Wu, L.H. Tang and D. Hu \\ School of Geography and Biological Information, \\ Nanjing University of Posts and Telecommunications, \\ Nanjing, Jiangsu, China \\ Corresponding author: X.H. Li \\ E-mail: lixh@njupt.edu.cn
}

Genet. Mol. Res. 14 (1): 2785-2792 (2015)

Received February 26, 2014

Accepted September 11, 2014

Published March 31, 2015

DOI http://dx.doi.org/10.4238/2015.March.31.8

\begin{abstract}
MicroRNAs (miRNAs) are a class of non-coding RNAs that play important roles in posttranscriptional regulation of target genes. miRNAs are involved in multiple biological processes by degrading targeted mRNAs or repressing mRNA translation in various organisms. Their conserved nature in various organisms makes them a good source of new miRNA discovery using comparative genomic approaches. In the present study, conserved Nile tilapia (Oreochromis niloticus) miRNAs were identified using a bioinformatic strategy based on expressed sequence tag and genome survey sequence databases. A total of 21 new miRNAs were detected and were found to belong to 17 families. Using mature miRNA sequences as queries, potential targets for tilapia miRNAs were predicted using a local BLAST program and the miRanda software. Target proteins identified using miRanda and BLAST analyses included transcription factors and molecules important in metabolism, transportation, immunity, stress-related activity, growth, and development. These miRNAs and their targets in tilapia may increase the understanding of the role of
\end{abstract}


miRNAs in regulating the growth and development of tilapia.

Key words: Bioinformatic analysis; Oreochromis niloticus; MicroRNAs; Target genes

\section{INTRODUCTION}

MicroRNAs (miRNAs) are a growing family of endogenous, non-protein-coding sequences that are 20-25 nucleotides (nt) long. They are hairpin-derived RNAs that repress the expression of target genes, typically by binding to the 3'-untranslated region (UTR) of mRNA (Chen and Rajewsky, 2007). More than 60\% protein-coding genes are computationally predicted to be targets based on base-pairing between the 3'-UTR and the 5'-seed region of the miRNA (Friedman et al., 2009). Numerous studies have demonstrated that miRNAs have important roles in biological functions, such as cell proliferation, differentiation, growth, development, carcinogenesis, responses to viral infection, and immune and stress responses in various organisms (Bartel, 2004; Blenkiron and Miska, 2007; Urbich et al., 2008).

In recent years, an increasing number of miRNAs have been identified and deposited in the major miRNA databases. Although hundreds of miRNA have been identified, only a very small number of fish miRNAs have been discovered and functionally identified. Several approaches are used to identify new miRNAs in fish. Direct cloning is a classic method that has enabled the identification of miRNAs in zebrafish (Kloosterman et al., 2006) and rainbow trout (Ramachandra et al., 2008). However, it is difficult to clone low-abundance miRNAs because all miRNAs have similar secondary hairpin structures and many of these structures are evolutionarily conserved (Ambros et al., 2003). Currently, high-throughput sequencing and computational methods have been commonly used to identify new miRNAs in fish. Using the small RNA deep sequencing protocol with bioinformatic analysis, known or new miRNAs were identified in zebrafish (Soares et al., 2009), medaka (Li et al., 2010), Atlantic halibut (Bizuayehu et al., 2013), commom carp (Zhu et al., 2012), and catfish (Xu et al., 2013). However, computational strategies based on expressed sequence tags (EST) and genome survey sequences (GSS), has been successful for identifying new miRNAs (mature and precursor) in fish (Barozai, 2012).

Nile tilapia (Oreochromis niloticus) is an economically important species in freshwater aquaculture. With its fast growth rate and ability to adapt to a wide range of culture conditions, tilapia is one of the most widely farmed fish in the world. Because miRNA is involved in growth and resistance to environmental stresses, several studies have attempted to identify miRNAs related to muscle growth, regulation of osmotic stress, and salt tolerance (Huang et al., 2012; Yan et al., 2013a). The full-miRNA transcriptome in muscle tissue revealed significant differences in miRNA expression between fast-growing and control strains of tilapia (Huang et al., 2012). miR-206, a muscle-specific miRNA, regulates the growth of teleost tilapia $(O$. niloticus) by regulating insulin-like growth factor 1 gene expression (Yan et al., 2013a). MyoD is a helix-loop-helix protein that regulates myogenesis, and miR-203b expression is negatively correlated with MyoD expression in tilapia (Yan et al., 2013b). In addition, miR-429 and miR-30c were found to regulate osmotic stress transcription factor 1 and salt tolerance in tilapia, respectively (Yan et al., 2012). Therefore, miRNAs play very important roles in biological processes in tilapia. However, the sequences of tilapia miRNAs, particularly precursor sequences, have not been thoroughly examined.

In this study, all known fish miRNAs from Danio rerio, Takifugu rubripes, Oryzias 
latipes, Tetraodon nigroviridis, and Cyprinus carpio in mirBase were used to search for conserved tilapia miRNA homologs in the EST and GSS databases. A total of 21 potential miRNAs were identified and the target sequences of these miRNAs were predicted. The results will provide information for miRNA studies of tilapia and other fish species.

\section{MATERIAL AND METHODS}

\section{Databases of miRNAs, EST, GSS, and mRNA sequences and software}

To search for potential miRNAs, a total of 675 previously identified miRNAs and their precursor sequences from 5 fish, including $D$. rerio, T. rubripes, O. latipes, T. nigroviridis, and C. carpio were obtained from miRBase (Release 19.0, September 2012; http:// microrna.sanger.ac.uk). These miRNAs were defined as a reference set of miRNA sequences. To avoid the identification of overlapping miRNAs, repeated sequences were removed and the remaining sequences were used as an miRNA reference. The $O$. niloticus EST, GSS, and mRNA databases were obtained from the NCBI GenBank nucleotide databases. The comparative BLAST 2.2.28 software was downloaded from the NCBI GenBank (http://www.ncbi.nlm. nih.gov/BLAST/).

\section{Prediction of potential miRNAs and their precursors}

All non-redundant mature miRNA sequences were used as BLAST queries against the Nile tilapia EST and GSS databases. The initial candidate tilapia miRNAs with 0-3 mismatches in all known fish mature miRNA sequences were predicted using the local BLAST 2.2.28. Additionally, $80 \mathrm{bp}$ of bilateral flanking sequences from the candidate miRNAs in the ESTs and GSS were extracted and their secondary structures were predicted using the webbased Mfold 3.1 software (http://www.bioinfo.rpi.edu/applications/mfold/) (Zuker, 2003). A sequence was considered to be a candidate miRNA precursor if the hairpin structure satisfied the criteria described by Fu et al. (2011): 1) the minimum free energy $(\Delta \mathrm{G})$ is $\leq-15 \mathrm{kcal} / \mathrm{mol}$; 2 ) the stem region includes at least $80 \%$ mature miRNA; 3 ) the number of allowed errors in 1 bulge is $\leq 18 \mathrm{bp}$; 4) the hairpin is $>53 \mathrm{bp}$ long; 5) the loop region is $<22 \mathrm{bp}$; and 6 ) the number of mismatches between the miRNA and the anti-stem sequence is $\leq 6 \mathrm{bp}$.

\section{Prediction of miRNA targets}

The $O$. niloticus miRNA targets were predicted using the miRanda program, which is publicly available (http://www.microrna.org/microrna/home.do). The parameters of miRanda were set as follows: the score was set to $\geq 100$ and the free energy was set to $\leq-20 \mathrm{kcal} / \mathrm{mol}$. In addition, another strategy was also used to predict potential target sequences of miRNAs. First, mature miRNAs identified in the present study were used to search for antisense hits in the reference RNA sequences (refseq_rna) of the tilapia (taxid: 8128). Subsequently, to predict target sequences, mRNA sequences exhibiting perfect or near perfect complementarity with corresponding miRNAs were analyzed using RNAhybrid (http://bibiserv.techfak.uni-bielefeld. de/rnahybrid), a miRNA target detection software program. The criteria for target prediction allowed up to 2 mismatches in the seed sequence, with 14 as a minimum number of paired-up bases in a heteroduplex and -25 as a maximum folding energy for a heteroduplex ( $\mathrm{kcal} / \mathrm{mol})$. 


\section{RESULTS AND DISCUSSION}

\section{Identification of potential miRNAs in $O$. niloticus}

Because most mature miRNAs are evolutionarily conserved between species, a comparative genetic strategy provides a powerful approach for predicting the existence of new miRNAs in other species. In this study, a total of 21 potential miRNAs were identified after homology searching using all previously identified fish miRNAs against the O. niloticus databases of 120,962 ESTs and 69,200 GSS. Thirteen miRNAs were identified in the EST database and 8 in the GSS database (Table 1). The miRNAs belonged to 17 families, including oni-let-7, mir-1, -22, -27, -31, -34, -133, -144, -153, -222, -301, and -429. In the let-7 family, 4 miRNAs were identified, followed by miR-182 and -199 with 2 miRNAs in each. The other 15 miRNA families had only 1 member each. Oni-let-7a-5 and oni-let-7e were found in the same EST (GR657356).

The lengths of the newly identified mature miRNAs varied from 20-24 nt, and were typically 22 nucleotides in length, except miR-31 (21 nt), miR-144 (20 nt), miR199-3 (23 nt), and miR-222 (24 nt). Their precursor miRNA lengths ranged from 66$122 \mathrm{nt}$. The location of mature miRNA sequences in the identified miRNAs also showed diversity, with sequences of let-7-i, miR-133, miR-182-a, miR-182-b, miR199-3a-2, miR199-1, miR-204, miR-222, miR-34, miR-27-a, miR-301-c, and miR-31 located at the 5'end of the pre-miRNAs and all others at the 3 '-end. There was a larger number of mature miRNAs located at the 5'-end of the pre-miRNA than at the 3 '-end (13:8). The A+U content of the predicted pre-miRNAs ranged from 35.6 to $59.3 \%$. Minimal folding-free energy $(\Delta \mathrm{G})$ of miRNA precursors varied from $23.30-46.70 \mathrm{kcal} / \mathrm{mol}$. The minimum folding-free energy index is a useful criterion for distinguishing miRNAs from other types of coding or non-coding RNAs. In our study, minimum folding-free energy index of the pre-miRNAs averaged 0.81 , which is significantly higher than that of tRNAs (0.64), rRNAs (0.59), and mRNAs (0.62-0.66) (Zhang et al., 2006).

\section{Prediction of miRNA targets}

miRNAs are involved in a diverse range of biological processes such as posttranscriptional regulation of target genes. In plants, miRNAs bind perfectly or near-perfectly to their targets and many of miRNAs have relatively few targets. In contrast to plant miRNAs, animal miRNAs bind to target mRNAs through imperfect complementary sequences that are typically located at the 3'-UTRs, leading to translational repression or transcript degradation. Complementarity to the core region (positions 1-10) of an miRNA is often sufficient for effective regulation in animals. Therefore, 1 miRNA can affect transcript and protein levels of hundreds of targets in animals (Ambros, 2004). Because short-complementary sequence stretches may be interrupted by gaps or mismatches, bioinformatic prediction of miRNA targets in animals is more complicated than in plants. Thus, 2 bioinformatic approaches were used to predict miRNA targets in this study.

Using the identified miRNA sequences as BLAST queries, 8 target genes for 5 miRNAs (including let-7b, mir-1, mir-153b, mir-182, and mir-429) were identified in the 
Identification of Nile tilapia miRNAs and target genes

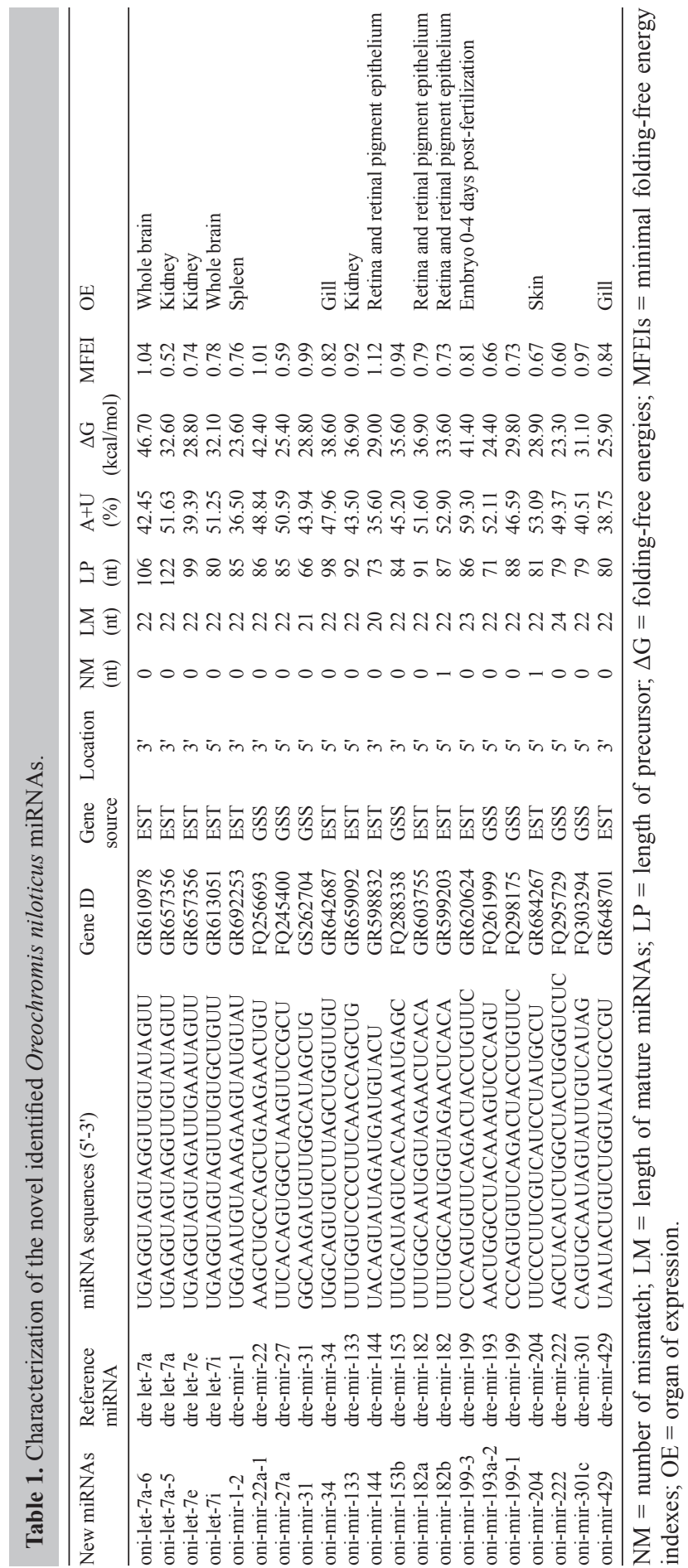


tilapia mRNA database (Table 2). Target genes and miRNAs showed a high level of sequence complementarity. mir- 1 was found to target the tropomyosin alpha- 4 chain, stromal interaction molecule 1, and dolichol-phosphate mannosyltransferase. Tropomyosin alpha-4 chain plays a central role, in association with the troponin complex, in the calcium-dependent regulation of vertebrate striated muscle contraction (Crabos et al., 1991). mir-1 expression in the muscle modulates cardiac and skeletal muscle proliferation and differentiation in zebrafish (Mishima et al., 2009) and humans (Koutsoulidou et al., 2011). Moreover, Nile tilapia mir-1 expression has been observed in skeletal muscle (Huang et al., 2012). This suggests that mir-1 plays important roles in modulating muscle contraction in tilapia.

\begin{tabular}{llll}
\multicolumn{2}{c}{ Table 2. Potential targets of the miRNAs in Nile tilapia by BLAST. } \\
\hline miRNA & Targeted protein & Target function & Target genes ID \\
\hline oni-let-7b & relA-associated inhibitor-like & Regulation of apoptosis and transcription & XM_003459126 \\
oni-mir-1 & Tropomyosin alpha-4 chain-like & Muscle contraction & XM_003439846 \\
& Stromal interaction molecule 1-like & Ion transport & XM_003455939 \\
oni-mir-153b & Dolichol-phosphate mannosyltransferase-like & Metabolism & XM_003456960 \\
& Apelin receptor B-like & Immunity response & XM_003444311 \\
oni-mir-182 & Long-chain fatty acid transport protein 4-like & Fatty acid transport & XM_003444353 \\
& ATPase, aminophospholipid transporter (APLT), & ATP binding & XM_003445257 \\
oni-mir-429 & class I, type 8A, member 1 & & \\
& E3 ubiquitin-protein ligase RNF180-like & Protein modification & XM_003440070 \\
\hline
\end{tabular}

BLAST only identifies target mRNAs with perfect or near-perfect complementary. In order to identify target genes with partial complementary, we used the miRanda software to predict other target genes of tilapia miRNAs. miRanda is one of the earliest-developed large-scale target prediction algorithms for vertebrates and is often used to predict miRNA target genes in fish (Chen et al., 2005). Because the 3'-UTRs of many predicted mRNAs could not be identified, they were not used in the miRanda prediction. A total of 24 targets for the 15 tilapia miRNAs were predicted in the Nile tilapia mRNA sequence (Table 3). According to the information provided by NCBI, the identified miRNA targets were classified as transcription factors or molecules important in metabolism, signaling, transport, immunity, stress responses, reproduction related, growth and development, and osmoregulation. These groups, except for reproduction-related molecules, have previously been identified as targets of miRNAs by experimental and/or computational approaches in animals (Barozai, 2012; Zhu et al., 2012). In addition, an miRNA can be complementary to multiple targets; a target can be complementary to several miRNAs correspondingly according to the prediction results. For example, 6 sequences were detected as targets of let$7 \mathrm{e}$, including the cytochrome $\mathrm{P} 4501 \mathrm{C} 1$ protein, the heat-shock protein 70, type I estrogen receptor, prolactin, 11-beta hydroxysteroid dehydrogenase type 2, and thrombospondin-1a. Additionally, oni-let-7a, oni-let-7e, and oni-mir-133 were all predicted to be regulatory miRNAs of the heat-shock protein 70 gene. These results suggest that an miRNA may have multiple different mRNA targets, and a target may similarly be targeted by multiple miRNAs in Nile tilapia. Similar findings were reported by many groups in different animal species (Rajewsky, 2006). Gaining insight into the Nile tilapia miRNA targets will increase the understanding of the range of miRNA expression regulation and more coherently describe the functional importance of miRNAs. 
Table 3. Potential targets of the miRNAs in Nile tilapia by miRanda program.

\begin{tabular}{|c|c|c|c|c|c|c|}
\hline New miRNAs & Target gene ID & Gene name & Position & Function & Energy & Score \\
\hline \multirow[t]{4}{*}{ oni-let-7a } & EU816596 & Heat-shock protein 70 & 2080 to 2101 & Stress responses & 21.31 & 112 \\
\hline & AY725227 & Liver hepcidin propeptide & 430 to 454 & Immunity & 22.30 & 110 \\
\hline & FJ455498 & Thrombospondin-1a & 4173 to 4197 & Stress responses & 21.02 & 103 \\
\hline & EU827279 & 3beta-hydroxysteroid dehydrogenase type I & 1883 to 1903 & Metabolism & 20.32 & 105 \\
\hline \multirow[t]{6}{*}{ oni-let-7e } & HQ829969 & Cytochrome $\mathrm{P} 4501 \mathrm{C} 1$ protein & 2135 to 2161 & Metabolism & -21.15 & 113 \\
\hline & EU816596 & Heat-shock protein 70 & 2080 to 2101 & Stress responses & -21.00 & 118 \\
\hline & U75604 & Type I estrogen receptor & 3728 to 3749 & Reproduction & -22.68 & 119 \\
\hline & M27010 & Prolactin & 870 to 893 & Osmoregulation & -20.20 & 109 \\
\hline & AY190043 & 11-beta hydroxysteroid dehydrogenase type 2 & 2145 to 2172 & Metabolism & -20.48 & 105 \\
\hline & FJ455498 & Thrombospondin-1a & 4173 to 4197 & Stress responses & -21.14 & 108 \\
\hline oni-let-7i & GQ214535 & Inducible cAMP early repressor & 484 to 508 & Transcription factor & -22.53 & 101 \\
\hline oni-mir-1-2 & AF247822 & Decorin & 1959 to 1983 & Fibril formation & -20.78 & 114 \\
\hline \multirow[t]{3}{*}{ oni-mir-133 } & EU816596 & Heat-shock protein 70 & 1853 to 1880 & Stress responses & -23.06 & 102 \\
\hline & HQ829968 & Cytochrome P450 1B1 protein & 1918 to 1941 & Metabolism & -22.09 & 100 \\
\hline & FJ455498 & Thrombospondin-1a & 4350 to 4371 & Stress responses & -23.48 & 103 \\
\hline oni-mir-153b & U75605 & Type II estrogen receptor & 4066 to 4090 & Reproduction & -21.28 & 100 \\
\hline oni-mir-199-3 & AF503208 & Glutamine synthetase & 1676 to 1696 & Metabolism & -23.95 & 108 \\
\hline \multirow[t]{2}{*}{ oni-mir-199-1 } & AY725227 & Liver hepcidin propeptide & 397 to 421 & Immunity & -21.57 & 107 \\
\hline & U75604 & Type I estrogen receptor & 4169 to 4190 & Reproduction & -20.16 & 107 \\
\hline \multirow[t]{3}{*}{ oni-mir-222 } & JN860432 & Type I estrogen receptor & 4404 to 4429 & Reproduction & -23.56 & 115 \\
\hline & U75604 & 11-beta hydroxysteroid dehydrogenase type 2 & 1143 to 1165 & Metabolism & -25.46 & 100 \\
\hline & AY190043 & Orphan nuclear receptor Dax-1 & 1721 to 1745 & Development & -27.37 & 123 \\
\hline \multirow[t]{2}{*}{ oni-mir-22a-1 } & HQ829968 & Cytochrome $\mathrm{P} 450$ 1B1 protein & 2024 to 2047 & Metabolism & -20.09 & 101 \\
\hline & AY149606 & DM-related transcriptional factor & 1588 to 1606 & Transcription factor & -24.08 & 102 \\
\hline \multirow[t]{2}{*}{ oni-mir-34 } & AY428948 & R-spondin-1 precursor & 1270 to 1288 & Sensory transduction & -20.58 & 104 \\
\hline & FJ411252 & $\begin{array}{l}\text { ATP-binding cassette subfamily } \\
\text { G member } 4 \text { transporter protein }\end{array}$ & 2563 to 2584 & Transport & -21.26 & 100 \\
\hline & M26916 & Growth-hormone & 807 to 831 & Growth and development & -21.27 & 102 \\
\hline \multirow[t]{2}{*}{ oni-mir-301c } & FJ914655 & Glucose transporter type $14.5-\mathrm{kb}$ transcript & 4225 to 4251 & Transport & -20.52 & 111 \\
\hline & AB182646 & tilDmc1 & 1449 to 1470 & DNA repair & -22.86 & 128 \\
\hline \multirow[t]{2}{*}{ oni-mir-31 } & AY330215 & MRE-binding transcription factor-1S & 1367 to 1390 & Transcription factor & -21.55 & 106 \\
\hline & AB075952 & Elongation factor $1 \mathrm{a}$ & 1463 to 1485 & Transcription factor & -25.72 & 110 \\
\hline \multirow[t]{2}{*}{ oni-mir-429 } & JN860434 & Fibroblast growth factor $20 \mathrm{~b}$ & 1154 to 1177 & Growth factor & -21.50 & 102 \\
\hline & FJ455498 & Thrombospondin-1a & 3879 to 3904 & Stress responses & -20.94 & 103 \\
\hline
\end{tabular}

\section{ACKNOWLEDGMENTS}

Research supported by the Nanjing University of Posts and Telecommunications Project for New Faculty (\#NY211031) and the Nanjing University of Posts and Telecommunications Project for Construction of the Laboratory (\#10).

\section{REFERENCES}

Ambros V (2004). The functions of animal microRNAs. Nature 431: 350-355.

Ambros V, Lee RC, Lavanway A, Williams PT, et al. (2003). MicroRNAs and other tiny endogenous RNAs in C. elegans. Curr. Biol. 13: 807-818.

Barozai MY (2012). Identification and characterization of the microRNAs and their targets in Salmo salar. Gene 499: 163-168.

Bartel DP (2004). MicroRNAs: genomics, biogenesis, mechanism, and function. Cell 116: 281-297.

Bizuayehu TT, Fernandes JM, Johansen SD and Babiak I (2013). Characterization of novel precursor miRNAs using next generation sequencing and prediction of miRNA targets in Atlantic halibut. PLoS One 4: e61378.

Blenkiron C and Miska EA (2007). miRNAs in cancer: approaches, aetiology, diagnostics and therapy. Hum. Mol. Genet. 16: 106-113.

Chen K and Rajewsky N (2007). The evolution of gene regulation by transcription factors and microRNAs. Nat. Rev. Genet. 8: 93-103. 
Chen PY, Manninga H, Slanchev K, Chien M, et al. (2005). The developmental miRNA profiles of zebrafish as determined by small RNA cloning. Genes Dev. 19: 1288-1293.

Crabos M, Yamakado T, Heizmann CW, Cerletti N, et al. (1991). The calcium binding protein tropomyosin in human platelets and cardiac tissue: elevation in hypertensive cardiac hypertrophy. Eur. J. Clin. Invest. 21: 472-478.

Friedman RC, Farth KK, Burge CB and Bartel DP (2009). Most mammalian mRNAs are conserved targets of microRNAs. Genome Res. 19: 92-105.

Fu Y, Shi Z, Wu M, Zhang J, et al. (2011). Identification and differential expression of microRNAs during metamorphosis of the Japanese flounder (Paralichthys olivaceus). PLoS One 6: e22957.

Huang CW, Li YH, Hu SY, Chi JR, et al. (2012). Differential expression patterns of growth-related microRNAs in the skeletal muscle of Nile tilapia (Oreochromis niloticus). J. Anim. Sci. 90: 4266-4279.

Kloosterman WP, Steiner FA, Berezikov E, de Bruijn E, et al. (2006). Cloning and expression of new microRNAs from zebrafish. Nucleic Acids Res. 9: 2558-2269.

Koutsoulidou A, Mastroyiannopoulos NP, Furling D, Uney JB, et al. (2011). Expression of miR-1, miR-133a, miR-133b and miR-206 increases during development of human skeletal muscle. BMC Dev. Biol. 11: 34.

Li S, Chan WC, Ho MR, Tsai KW, et al. (2010). Discovery and characterization of medaka miRNA genes by next generation sequencing platform. BMC Genomics 11 (Suppl 4): S8.

Mishima Y, Abreu-Goodger C, Staton AA, Stahlhut C, et al. (2009). Zebrafish miR-1 and miR-133 shape muscle gene expression and regulate sarcomeric actin organization. Genes Dev. 23: 619-632.

Rajewsky N (2006). microRNA target predictions in animals. Nat. Genet. 38: S8-S13.

Ramachandra RK, Salem M, Gahr S, Rexroad CE 3rd, et al. (2008). Cloning and characterization of microRNAs from rainbow trout (Oncorhynchus mykiss): their expression during early embryonic development. BMC Dev. Biol. 8: 41.

Soares AR, Pereira PM, Santos B, Egas C, et al. (2009). Parallel DNA pyrosequencing unveils new zebrafish microRNAs. BMC Genomics 10: 195.

Urbich C, Kuehbacher A and Dimmeler S (2008). Role of microRNAs in vascular diseases, inflammation, and angiogenesis. Cardiovasc. Res. 79: 581-588.

Xu Z, Chen J, Li X, Ge J, et al. (2013). Identification and characterization of microRNAs in channel catfish (Ictalurus punctatus) by using Solexa sequencing technology. PLoS One 8: e54174.

Yan B, Guo JT, Zhao LH and Zhao JL (2012). MiR-30c: a novel regulator of salt tolerance in tilapia. Biochem. Biophys. Res. Commun. 425: 315-320.

Yan B, Zhu CD, Guo JT, Zhao LH, et al. (2013a). miR-206 regulates the growth of the teleost tilapia (Oreochromis niloticus) through the modulation of IGF-1 gene expression. J. Exp. Biol. 216: 1265-1269.

Yan B, Guo JT, Zhu CD, Zhao LH, et al. (2013b). miR-203b: a novel regulator of MyoD expression in tilapia skeletal muscle. J. Exp. Biol. 216: 447-451.

Zhang BH, Pan XP, Cox SB, Cobb GP, et al. (2006). Evidence that miRNAs are different from other RNAs. Cell Mol. Life Sci. 63: 246-254.

Zhu YP, Xue W, Wang JT, Wan YM, et al. (2012). Identification of common carp (Cyprinus carpio) microRNAs and microRNA-related SNPs. BMC Genomics 13: 413.

Zuker M (2003). Mfold web server for nucleic acid folding and hybridization prediction. Nucleic Acids Res. 31: $3406-$ 3415 . 